\title{
Enhanced Li-Ion Conductivity in Nanosized $\mathrm{Li}_{10} \mathrm{GeP}_{2} \mathrm{~S}_{12}$
}

James A. Dawson ${ }^{1,2, *}$ and M. Saiful Islam ${ }^{2, *}$

${ }^{1}$ Chemistry - School of Natural and Environmental Sciences, Newcastle University, Newcastle upon Tyne, NE1 7RU, UK

${ }^{2}$ Department of Chemistry, University of Bath, Bath, BA2 7AY, UK

*Corresponding authors. Emails: james.dawson@ newcastle.ac.uk; m.s.islam@bath.ac.uk

The discovery of the lithium superionic conductor $\mathrm{Li}_{10} \mathrm{GeP}_{2} \mathrm{~S}_{12}$ (LGPS) has led to significant research activity on solid electrolytes for high-performance and safe solid-state batteries. LGPS exhibits a remarkably high room-temperature Li-ion conductivity of $12 \mathrm{mS} / \mathrm{cm}$, comparable to that of the liquid electrolytes used in current Li-ion batteries. Here, we predict that nanosizing of LGPS can be used to further enhance its already outstanding Li-ion conductivity. By utilizing state-of-the-art nanoscale molecular dynamics techniques, we are able to simulate the Li-ion conductivities of nanocrystalline LGPS systems with average grain sizes from 10 to 2 $\mathrm{nm}$. Our results reveal that the Li-ion conductivity of LGPS increases with decreasing grain volume. For the smallest nanometric grain size, the Li-ion conductivity at room temperature is three times higher that of the bulk system. These findings reveal that nanosizing LGPS and related solid electrolytes could be an effective approach for enhancing their Li-ion conductivity. 
Solid-state batteries are currently at the forefront of the quest for next-generation energy storage technologies. The development of safe and energy dense solid-state batteries hinges on the replacement of the organic liquid electrolytes found in current commercial batteries with solid electrolytes ${ }^{1-5}$.

Following the first report ${ }^{6}$ of the lithium superionic conductor $\mathrm{Li}_{10} \mathrm{GeP}_{2} \mathrm{~S}_{12}$ (LGPS), this material has attracted considerable attention as a solid electrolyte for solid-state batteries ${ }^{7-26}$. This interest primarily results from its exceptional room temperature Li-ion conductivity of 12 $\mathrm{mS} / \mathrm{cm}$, matching that of current liquid electrolytes, and its excellent electrochemical performance ${ }^{6}$. The LGPS structural family therefore represents a crucial component in the development of solid-state batteries.

Nanostructured energy materials have attracted considerable interest because of the potential for unusual properties endowed by confining their dimensions ${ }^{27}$. However, while the ion transport mechanisms in bulk LGPS have been investigated ${ }^{7-9}$, this is not the case for grain boundary or nanocrystallite effects in LGPS ceramic samples. Grain boundary resistance in sulfide solid electrolytes is generally considered to be minimized compared to that of oxide systems $^{28-30}$. For LGPS, grain boundary resistance has been shown to only be significant below $250 \mathrm{~K}^{10,26}$.

Beyond LGPS, there are several studies concerning the microstructure and grain boundaries of various solid electrolytes in order to reduce interfacial resistance and improve ion transport ${ }^{31-38}$. The influence of nanosizing crystalline samples on Li-ion conductivity has been illustrated for sulfide ${ }^{38-40}$ and oxide ${ }^{41-43}$ solid electrolyte materials.

In this study, we show how reducing the size of LGPS crystallites to the nanoscale results in a substantial enhancement in Li-ion conductivity. Using a novel nanoscale molecular dynamics approach, we are able to directly simulate the Li-ion conductivity of LGPS as a 
function of its grain size. We find a clear trend of increasing Li-ion conductivity with decreasing grain size. Our findings represent a nanosizing approach for the enhancement of the superionic conductivity of LGPS and have wide implications for the optimization of solid electrolytes in general.

\section{Results}

Li-ion conductivity of bulk vs. nanocrystalline LGPS. To determine the influence of the bulk system versus nanosizing on LGPS, we first constructed cubic nanocrystalline systems each consisting of $\sim 100000$ ions with three different average grain volumes $\left(10,100\right.$ and $\left.1000 \mathrm{~nm}^{3}\right)$. These nanocrystals were then investigated using large-scale molecular dynamics (MD) with long simulation times of $<10 \mathrm{~ns}$, as detailed in the Methods section. We stress that the system sizes and timescales used in this work are orders of magnitude greater than those that can be achieved with ab initio MD.

Examples of the nanocrystals investigated in this study are given in Figure 1. Two unique nanocrystals were created for each grain volume and the results averaged. The variation between the two nanocrystals for each grain volume was minimal. The process used to construct the nanocrystals is described in the Methods section and has been recently applied to successful studies of $\mathrm{Na}_{3} \mathrm{PO}_{4}$ and $\mathrm{Na}_{3} \mathrm{PS}_{4}$ solid electrolytes ${ }^{28}$. 


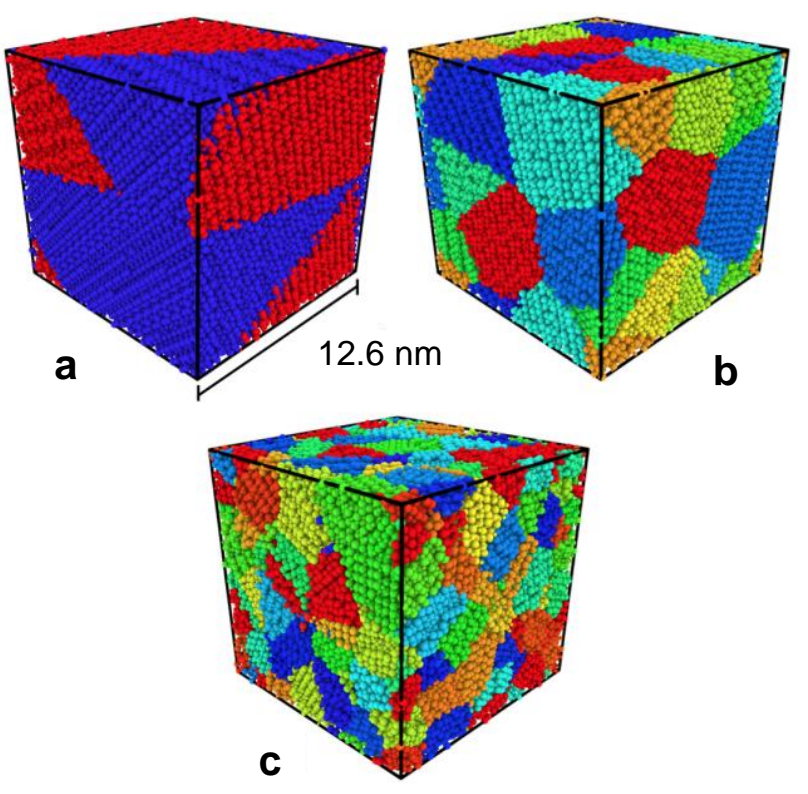

Fig. 1 Cubic nanocrystals of LGPS. a Nanocrystal containing 2 grains with an average grain volume of $1000 \mathrm{~nm}^{3}$. b Nanocrystal containing 20 grains with an average grain volume of 100 $\mathrm{nm}^{3}$. c Nanocrystal containing 200 grains with an average grain volume of $10 \mathrm{~nm}^{3}$. The grain volumes are simply determined by dividing the total nanocrystal volume by the total number of grains in that nanocrystal. Each color represents a unique grain.

The calculated Li-ion conductivities for bulk and nanocrystalline LGPS are plotted in Figure 2 along with data from experiment ${ }^{6,13}$. The calculated bulk conductivities are slightly underestimated compared to experimental values for polycrystalline $e^{6,13}$ and single-crystal ${ }^{26}$ LGPS but are in good agreement with previous ab initio MD (when extrapolated from high temperatures) $^{7}$ and classical MD simulations ${ }^{8}$.

The results in Figure 2 predict that the Li-ion conductivity of LGPS increases as a result of dramatically decreasing the grain volume, i.e., nanosizing. The highest conductivities are found for the smallest grain volume of $10 \mathrm{~nm}^{3}$ (with an average grain size of $\sim 2.15 \mathrm{~nm}$ ), with a value of $15.10 \mathrm{mS} \mathrm{cm}^{-1}$ obtained at $300 \mathrm{~K}$, which is higher than that found in the seminal study of Kamaya et al. $^{6}$ at the same temperature $\left(12 \mathrm{mS} \mathrm{cm}^{-1}\right)$ and indeed subsequent experimental studies of LGPS ${ }^{13}$. Moreover, this conductivity of $15.10 \mathrm{mS} \mathrm{cm}^{-1}$ is almost triple the 
conductivity calculated for the bulk (single crystal) system of $5.87 \mathrm{mS} \mathrm{cm}^{-1}$. It is noteworthy that the largest jump in conductivity between the different simulated systems occurs between the smallest grain volumes of 100 and $10 \mathrm{~nm}^{3}$.

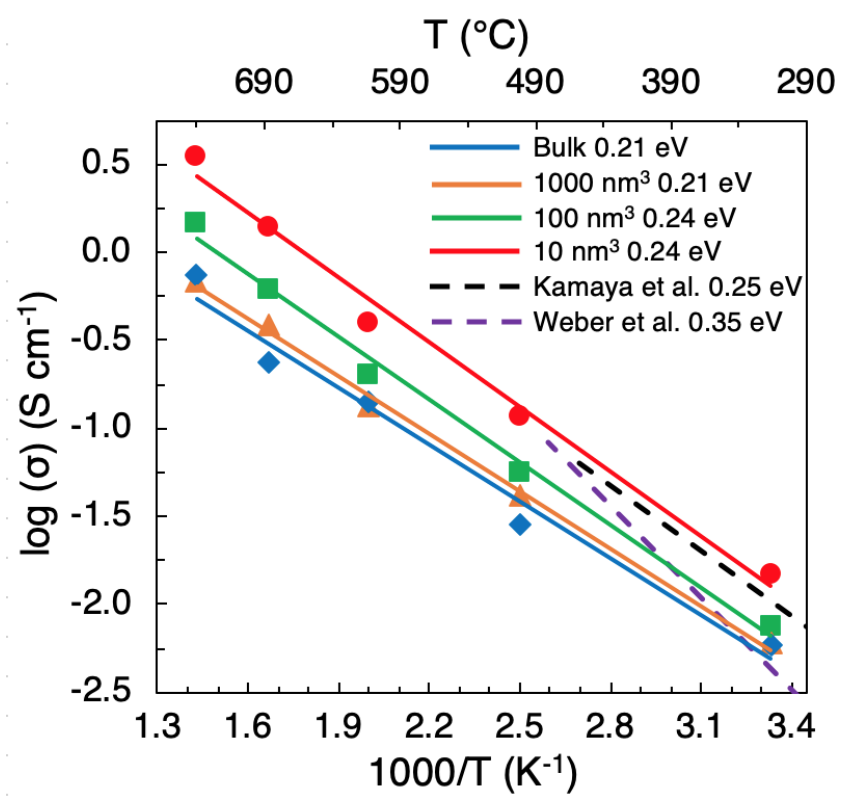

Fig. 2 Li-ion transport in bulk and nanocrystalline LGPS. Li-ion conductivities $(\sigma)$ and activation energies $\left(E_{\mathrm{a}}\right)$ for bulk and nanocrystalline LGPS (for three different grain volumes of 1000,100 and $10 \mathrm{~nm}^{3}$ ) compared to previous experimental ${ }^{6,13}$ studies.

The calculated activation energies $(0.21-0.24 \mathrm{eV})$ are all in excellent agreement with values derived from impedance $(0.22-0.35 \mathrm{eV})^{6,10,13,26}$ and NMR $(0.21 \mathrm{eV})^{10}$ measurements. While grain boundary resistance is often considered to be minimal in LGPS and its derivatives ${ }^{16,18}$, our results show that grain boundaries may actually become beneficial to Li-ion conduction in these materials when the grain size is sufficiently small $(<10 \mathrm{~nm})$.

Although our results present a clear prediction of the effects of nanosizing on $\mathrm{Li}$-ion transport in LGPS, we recognize that it remains highly challenging to synthesize LGPS particles with the nanoscale average grain sizes $(\sim 2-10 \mathrm{~nm})$ utilized in our simulations. Recent studies ${ }^{44,45}$ have reported the synthesis of LGPS with grain sizes of $\sim 100-1000 \mathrm{~nm}$; however, these sizes are still substantially larger than those in this study. Nevertheless, it is encouraging 
that nanocrystals of the related sulfide electrolyte $\mathrm{Li}_{3.25} \mathrm{P}_{0.95} \mathrm{~S}_{4}$ as small as $5 \mathrm{~nm}$ in an amorphous matrix have been reported ${ }^{40}$, and that a massive enhancement in Li-ion conductivity was observed for nanoporous $\mathrm{Li}_{3} \mathrm{PS}_{4}$ with average particle sizes of $80-100 \mathrm{~nm}^{38}$. In addition, it is important to bear in mind that sizes of $<10 \mathrm{~nm}^{3}$ are difficult to detect using XRD and instead appear as amorphous ${ }^{40,46}$. Therefore, it may be necessary to utilize other techniques, such as transmission electron microscopy, to observe such minute crystalline domains.

Given the similarities between their structural and grain boundaries properties, it is important to emphasize that the enhancement in ion conductivity found for LGPS as a result of nanosizing may also be applicable to many of its derivatives; these include Li9.54 $\mathrm{Si}_{1.74} \mathrm{P}_{1.44} \mathrm{~S}_{11.7} \mathrm{Cl}_{0.3}{ }^{17}$, an even faster Li-ion diffusing solid electrolyte, which warrants future investigation.

Ion transport pathways. It is known that the dimensionality of Li-ion transport within crystal structures of solid electrolyte materials is important for their ionic conductivity. The impact of the disorder caused by the grain boundaries on the Li-ion transport in LGPS can be observed by visualizing the $\mathrm{Li}$-ion trajectories during the simulations. Figure 3(a) shows the accumulated Li-ion trajectories for bulk LGPS at $300 \mathrm{~K}$. As reported in previous experimental and computational works ${ }^{6-8,13,26}$, the most facile Li-ion diffusion occurs in one-dimensional channels along the $c$ direction of the tetragonal structure, with diffusion also taking place in the $a b$ plane. This is also supported by our results in Figure 3(c), where the mean squared displacement (MSD) of Li ions is plotted for the three primary directions. We find that diffusion in the $c$ direction is approximately five-fold higher than in the $a b$ plane. Such anisotropic ionic conductivity is also observed from impedance measurements of single crystals of LGPS ${ }^{26}$. 
a
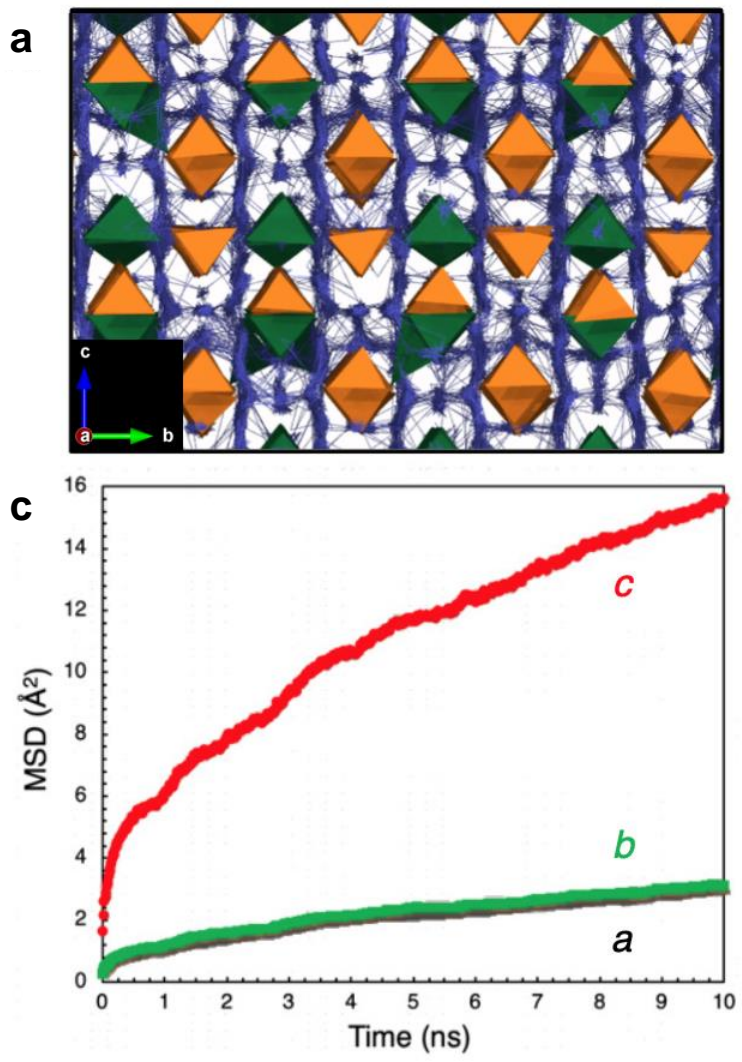

b

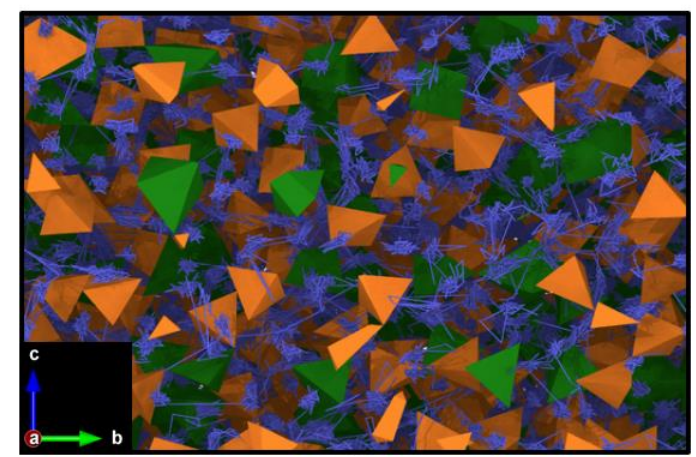

d

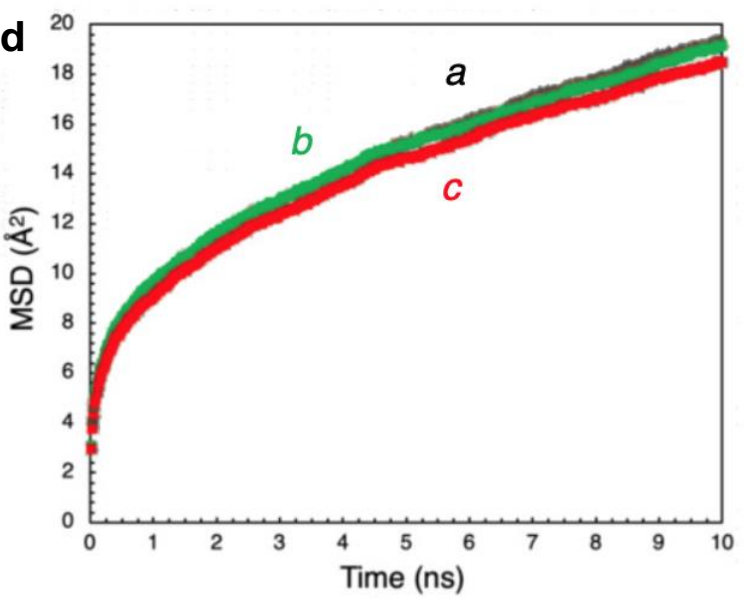

Fig. 3 Li-ion diffusion pathways in bulk and nanocrystalline LGPS. a Diffusion density plots of Li ions (blue) overlaid on $\mathrm{GeS}_{4}$ (green) and $\mathrm{PS}_{4}$ (orange) tetrahedra in bulk LGPS. b Diffusion density plots of Li ions in nanocrystalline LGPS with a grain volume of $10 \mathrm{~nm}^{3}$ at $300 \mathrm{~K}(2.4 \times 3.5 \mathrm{~nm}$ cross section). $\mathbf{c}$ MSD plots of Li-ion diffusion in bulk LGPS at $300 \mathrm{~K}$ for the $a, b$ and $c$ directions. $\mathbf{d}$ MSD plots of Li-ion diffusion in nanocrystalline LGPS with a grain volume of $10 \mathrm{~nm}^{3}$ at $300 \mathrm{~K}$.

Figure 3(b) presents the Li-ion trajectories for nanocrystalline LGPS with the smallest grain volume of $10 \mathrm{~nm}^{3}$ at $300 \mathrm{~K}$. In contrast to the well-defined Li-ion diffusion pathways for bulk LGPS, the diffusion pathways for nanosized LGPS are far more isotropic, indicating fast diffusion in all directions. This is further corroborated by the MSD plots given in Figure 3(d), where it is now clear that there is no longer a preference for Li-ion diffusion in the $c$ direction, with significant raising of the in-plane diffusion. The additional advantage of nanosizing is the 
short path length for Li-ion transport. Hence, the high concentration of grain boundaries in this nanocrystal allow the Li ions to readily diffuse in all directions, beyond the preferential diffusion pathways in the $c$ and $a b$ directions in bulk LGPS. Such isotropic conduction behavior allows lithium access through all surfaces of the LGPS particles.

Local structures and ion coordination. To investigate and understand the local structural factors that influence Li-ion transport in these nanocrystalline systems, we analyze the radial distribution functions (RDFs) of ion pairs in LGPS. Figure 4 shows a comparison of the RDFs for the Li-Li, Li-S, Ge-Ge and P-P pairs of the bulk versus nanocrystalline system (for the smallest grain volume of $10 \mathrm{~nm}^{3}$ ). Two key features emerge. First, the values beyond the first maximum peak for Li-Li indicate that the Li distribution is disordered in LGPS indicative of a superionic conductor. We note that the main maximum peaks of the bulk RDFs at 2.36 and 3.40 $\AA$ for Li-S and Li-Li, respectively, are in excellent agreement with the experimental structure ${ }^{6}$, as well as RDFs calculated using ab initio $\mathrm{MD}^{47}$.

Second, all the RDFs for the nanocrystals exhibit broader peaks especially after the first maximum peak and are associated with the increased disorder in these systems. By far the greatest difference in the nanocrystal compared to the bulk is found for the Ge-Ge and P-P RDFs, with significant peak broadening in the nanocrystal. This increased disorder agrees with the distinct differences found in the diffusion density plots and ion conduction pathways shown in Figures 3(a) and (b). The remaining RDFs for bulk and nanocrystalline LGPS show similar features and are given in Supplementary Figure 1. 

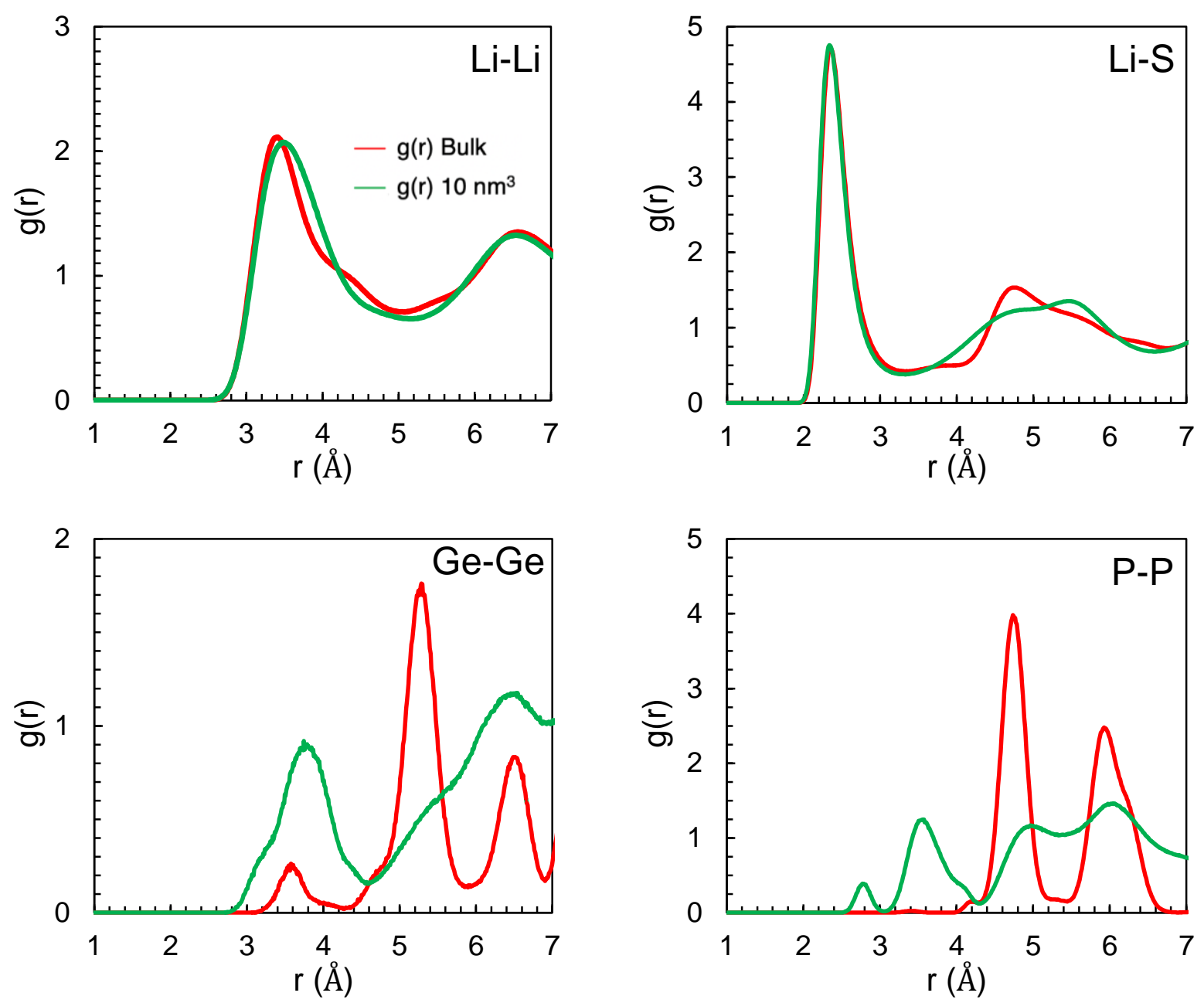

Fig. 4 Local structural differences between bulk and nanocrystalline LGPS. Radial distribution functions (RDFs) of Li-Li, Li-S, Ge-Ge and P-P RDFs (g(r)) for bulk (red) and nanocrystalline (green, grain volume of $10 \mathrm{~nm}^{3}$ ) LGPS at $300 \mathrm{~K}$.

To further illustrate the influence of ion coordination on the Li-ion transport of nanosized LGPS, we plot the difference in the Li-Li and Li-S coordination numbers between bulk $\left(\mathrm{n}(\mathrm{r})_{\text {bulk }}\right)$ and nanocrystalline $\left(10 \mathrm{~nm}^{3}, \mathrm{n}(\mathrm{r})_{\text {nano }}\right)$ LGPS, shown in Figure 5. In these plots, values above zero reflect higher coordination in the bulk compared to the nanocrystal, while values below zero represent reduced coordination in the nanocrystal.

The results in Figure 5 show that $\mathrm{Li}-\mathrm{Li}$ and $\mathrm{Li}-\mathrm{S}$ coordination decreases by one to two for distances of $>4.6 \AA$. We propose that this decrease in lithium coordination (or 
undercoordination) contributes to the high Li-ion conductivity observed in the nanocrystalline systems. The changes in local structure resulting from the nanosizing of LGPS, as well as the significant increase in its grain boundary-to-bulk ratio, are clearly involved in the enhancement of Li-ion conductivity in this material.

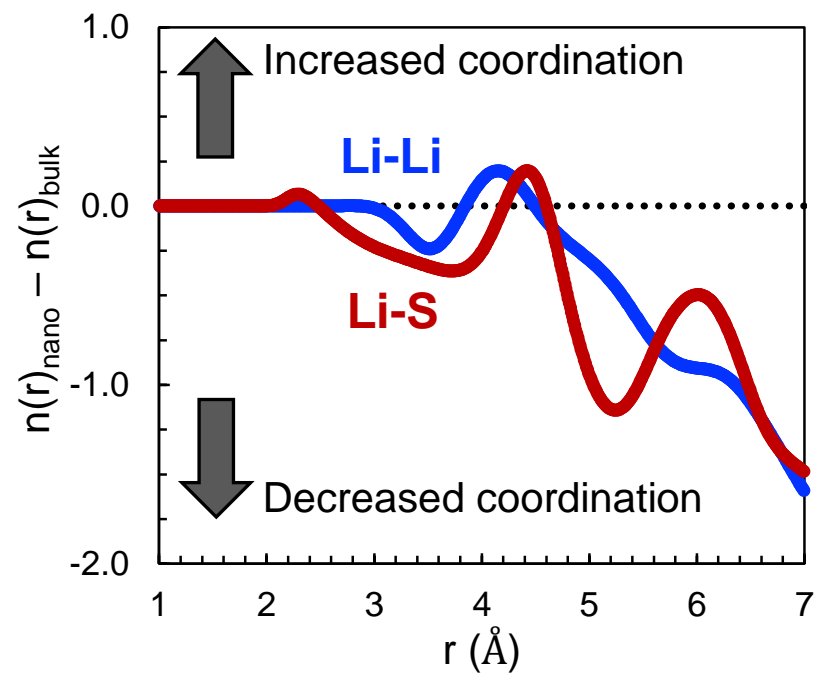

Fig. 5 Ion coordination differences between bulk and nanocrystalline LGPS. Plots of difference between Li-Li and Li-S coordination numbers in bulk $\left(\mathrm{n}(\mathrm{r})_{\text {bulk }}\right)$ and nanocrystalline $\left(10 \mathrm{~nm}^{3}, \mathrm{n}(\mathrm{r})_{\text {nano }}\right)$ LGPS as a function of $\mathrm{r}$. Positive and negative values represent increased and decreased coordination, respectively.

We reported similar findings in a previous $\mathrm{study}^{28}$, where the decrease in Na coordination (undercoordination) resulted in an enhancement in Na-ion transport for polycrystalline $\mathrm{Na}_{3} \mathrm{PS}_{4}$. However, it is noteworthy that the local structural changes in LGPS in this work are more substantial than those found for $\mathrm{Na}_{3} \mathrm{PS}_{4}$.

Discussion. In summary, the substantial effects of nanosizing the promising solid electrolyte material, $\mathrm{Li}_{10} \mathrm{GeP}_{2} \mathrm{~S}_{12}$, are reported. Through a nanoscale simulation approach, we have demonstrated that moving from bulk LGPS to the nanoscale $(<10 \mathrm{~nm})$ results in a large enhancement in Li-ion conductivity; this effect is attributed to changes in the ion conduction 
pathways and local environment. In bulk LGPS, Li-ion transport is anisotropic with faster diffusion in the $c$ direction than within the $a b$ plane. In contrast, in LGPS nanocrystals with nanometric grain sizes, there is a switch to isotropic Li-ion conduction with fast transport in all directions. We also find greater local structural disorder and a decrease in Li coordination (or undercoordination) with decreasing grain volume, which also facilitates the high Li-ion conductivity. Despite the challenges of nanoscale synthesis, these findings open up an alternative nanosizing approach to enhance the Li-ion conductivity of LGPS and suggests a design route for further experimental work.

\section{Methods}

Molecular dynamics simulations. The MD simulations in this work are based on established techniques and have been widely used to determine the ion transport properties in a wide variety of Liand Na-ion solid electrolytes ${ }^{28,30,35,48-52}$. The calculations were performed using the LAMMPS code $^{53}$ with long MD runs of $10 \mathrm{~ns}$ were completed using a time step of $1 \mathrm{fs}$ and supercells of $~ 100000$ ions for both the bulk (single crystal) and nanocrystal systems. The bulk crystal structure of LGPS was obtained from the Materials Project ${ }^{54}$, with the disordered site occupancies ordered using the same method used in previous studies ${ }^{55}$.

Simulations were carried out for a temperature range of 300-700 K at intervals of $100 \mathrm{~K}$ using the NVT ensemble with a Nose-Hoover thermostat ${ }^{56}$, with initial equilibration performed using the NPT ensemble for $\sim 2 \mathrm{~ns}$. Self-diffusion data for Na were obtained from an MSD analysis according to $\left\langle r_{i}^{2}(t)\right\rangle=6 D_{\mathrm{Li}} t$

where $\left\langle r_{i}^{2}(t)\right\rangle$ is the MSD, $D_{\mathrm{Li}}$ is the diffusion coefficient for $\mathrm{Li}$ and $t$ is time. The diffusion data were then converted to conductivities $(\sigma)$ using the Nernst-Einstein relationship

$$
\frac{}{D_{L i}} H_{\mathrm{R}} \frac{n q^{2}}{k T}
$$


where $n$ is the number of $\mathrm{Li}$ ions per unit volume, $q$ is the electron charge, $k$ is the Boltzmann constant, $T$ is the temperature and $H_{\mathrm{R}}$ is the Haven ratio, which is set to 3 in our calculations based on the ab initio MD simulations of $\mathrm{He}$ et al. ${ }^{55}$.

Potential model. The potential model of Kim et al. ${ }^{49}$ developed for $\left(\mathrm{Li}_{2} \mathrm{~S}\right)_{0.75}\left(\mathrm{P}_{2} \mathrm{~S}_{5}\right)_{0.25}$ and $\mathrm{Li}_{3} \mathrm{PS}_{4}$ was used for the simulations in this work, with the addition of a newly derived Morse potential for the GeS interaction in LGPS (using the GULP $\operatorname{code}^{57}$ ). This potential was successfully used to study Li-ion transport in crystalline and glassy thiosulphate solid electrolytes ${ }^{49}$. The charge of Ge in the potential model was determined using Bader charge analysis ${ }^{58}$ through density functional theory calculations with the VASP code ${ }^{59}$. The projector augmented wave method $^{60}$ and the PBEsol exchange-correlation functional ${ }^{61}$ were employed with a plane-wave cut-off energy of $520 \mathrm{eV}$ and a k-point mesh spacing smaller than $0.05 \AA^{-1}$. The full potential model and atomic charges are tabulated in Supplementary Table 1. The calculated lattice parameters for LGPS of $a=8.501 \AA$ and $c=12.822 \AA$ are in good agreement with those obtained using X-ray diffraction of $a=8.718 \AA$ and $c=12.635 \AA^{6}$.

Construction of nanocrystalline models. The nanocrystal models used in this study were constructed using Voronoi tessellations, as employed in the Atomsk program ${ }^{62}$, in which nodes are introduced at given positions inside the simulation box that are then linked with their neighboring nodes. The normals to these links are then found and these define the contours of the randomly orientated grains, that is, the grain boundaries in this study. Unit cells are then placed at the nodes and are expanded in three dimensions. The final nanocrystal is then obtained after the unit cells have been expanded and cut into the respective grains. Cubic nanocrystals with dimensions of $126 \times 126 \times 126 \AA^{3}$ were used. Nanocrystals with 2, 20 and 200 grains (equivalent to grain volumes of 1000, 100 and $10 \mathrm{~nm}^{3}$, respectively) were used to investigate the effects of nanosizing on Li-ion transport in LGPS. MD simulations were carried out on two different random polycrystals for each grain volume and the data were averaged. 


\section{Acknowledgements}

The authors gratefully acknowledge the EPSRC Programme Grant "Enabling next generation lithium batteries" (EP/M009521/1) and the MCC/Archer consortium (EP/L000202/1). JAD also gratefully acknowledges Newcastle University for funding through a Newcastle Academic Track (NUAcT) Fellowship.

\section{References}

1. Famprikis, T., Canepa, P., Dawson, J. A., Islam, M. S. \& Masquelier, C. Fundamentals of inorganic solid-state electrolytes for batteries. Nat. Mater. 18, 1278-1291 (2019).

2. Zhang, Z. et al. New horizons for inorganic solid state ion conductors. Energy Environ. Sci. 11, 1945$1976(2018)$.

3. Manthiram, A., Yu, X. \& Wang, S. Lithium Battery Chemistries Enabled by Solid-State Electrolytes. Nat. Rev. Mater. 2, 16103 (2017).

4. Bachman, J. C. et al. Inorganic solid-state electrolytes for lithium batteries: Mechanisms and properties governing ion conduction. Chem. Rev. 116, 140-162 (2016).

5. Janek, J. \& Zeier, W. G. A solid future for battery development. Nat. Energy 1, 16141 (2016).

6. Kamaya, N. et al. A lithium superionic conductor. Nat. Mater. 10, 682-686 (2011).

7. Mo, Y., Ong, S. P. \& Ceder, G. First principles study of the $\mathrm{Li}_{10} \mathrm{GeP}_{2} \mathrm{~S}_{12}$ lithium super ionic conductor material. Chem. Mater. 24, 15-17 (2012).

8. Adams, S. \& Prasada Rao, R. Structural requirements for fast lithium ion migration in $\mathrm{Li}_{10} \mathrm{GeP}_{2} \mathrm{~S}_{12}$. J. Mater. Chem. 22, 7687-7691 (2012).

9. Ong, S. P., Mo, Y., Richards, D. \& Miara, L. Phase Stability, Electrochemical Stability and Ionic conductivity of the $\mathrm{Li}_{10 \pm 1} \mathrm{MP}_{2} \mathrm{X}_{12}(\mathrm{M}=\mathrm{Ge}, \mathrm{Si}, \mathrm{Sn}, \mathrm{Al}$ or $\mathrm{P}$, and $\mathrm{X}=\mathrm{O}, \mathrm{S}$ or $\mathrm{Se})$ family of superionic conductors. Energy Environ. Sci. 12, 148-156 (2013).

10. Kuhn, A., Duppel, V. \& Lotsch, B. V. Tetragonal $\mathrm{Li}_{10} \mathrm{GeP}_{2} \mathrm{~S}_{12}$ and $\mathrm{Li}_{7} \mathrm{GePS}_{8}-$ exploring the Li ion dynamics in LGPS Li electrolytes. Energy Environ. Sci. 6, 3548-3552 (2013). 
11. Kuhn, A. et al. A new ultrafast superionic Li-conductor: Ion dynamics in $\mathrm{Li}_{11} \mathrm{Si}_{2} \mathrm{PS}_{12}$ and comparison with other tetragonal LGPS-type electrolytes. Phys. Chem. Chem. Phys. 16, 14669-14674 (2014).

12. Han, F., Gao, T., Zhu, Y., Gaskell, K. J. \& Wang, C. A battery made from a single material. $A d v$. Mater. 27, 3473-3483 (2015).

13. Weber, D. A. et al. Structural insights and 3D diffusion pathways within the lithium superionic conductor $\mathrm{Li}_{10} \mathrm{GeP}_{2} \mathrm{~S}_{12}$. Chem. Mater. 28, 5905-5915 (2016).

14. Han, F., Zhu, Y., He, X., Mo, Y. \& Wang, C. Electrochemical stability of $\mathrm{Li}_{10} \mathrm{GeP}_{2} \mathrm{~S}_{12}$ and $\mathrm{Li}_{7} \mathrm{La}_{3} \mathrm{Zr}_{2} \mathrm{O}_{12}$ solid electrolytes. Adv. Energy Mater. 6, 1501590 (2016).

15. Hori, S., Suzuki, K., Hirayama, M., Kato, Y. \& Kanno, R. Lithium superionic conductor $\mathrm{Li}_{9.42} \mathrm{Si}_{1.02} \mathrm{P}_{2.1} \mathrm{~S}_{9.96} \mathrm{O}_{2.04}$ with $\mathrm{Li}_{10} \mathrm{GeP}_{2} \mathrm{~S}_{12}$-type structure in the $\mathrm{Li}_{2} \mathrm{~S}-\mathrm{P}_{2} \mathrm{~S}_{5}-\mathrm{SiO}_{2}$ pseudoternary system: Synthesis, electrochemical properties, and structure-composition relationships. Front. Energy Res. 4, 38 (2016).

16. Bron, P., Dehnen, S. \& Roling, B. $\mathrm{Li}_{10} \mathrm{Si}_{0.3} \mathrm{Sn}_{0.7} \mathrm{P}_{2} \mathrm{~S}_{12}-$ a low- cost and low-grain-boundaryresistance lithium superionic conductor. J. Power Sources 329, 530-535 (2016).

17. Kato, Y. et al. High-power all-solid-state batteries using sulfide superionic conductors. Nat. Energy 1, 16030 (2016).

18. Sun, Y. et al. Oxygen substitution effects in $\mathrm{Li}_{10} \mathrm{GeP}_{2} \mathrm{~S}_{12}$ solid electrolyte. J. Power Sources 324, 798-803 (2016).

19. Sun, Y., Suzuki, K., Hori, S., Hirayama, M. \& Kanno, R. Superionic conductors: $\operatorname{Li}_{10+\delta}\left[\mathrm{Sn}_{\mathrm{y}} \mathrm{Si}_{1-}\right.$ y $]_{1+\delta} \mathrm{P}_{2-\delta} \mathrm{S}_{12}$ with a $\mathrm{Li}_{10} \mathrm{GeP}_{2} \mathrm{~S}_{12}$-type structure in the $\mathrm{Li}_{3} \mathrm{PS}_{4}-\mathrm{Li}_{4} \mathrm{SnS}_{4}-\mathrm{Li}_{4} \mathrm{SiS}_{4}$ quasi-ternary system. Chem. Mater. 29, 5858-5864 (2017).

20. Gao, Y. et al. Salt-based organic-inorganic nanocomposites: Towards a stable lithium metal/ $/ \mathrm{Li}_{10} \mathrm{GeP}_{2} \mathrm{~S}_{12}$ solid electrolyte interface. Angew. Chem. Int. Ed. 57, 13608-13612 (2018).

21. Oh, K. et al. Native defects in $\mathrm{Li}_{10} \mathrm{GeP}_{2} \mathrm{~S}_{12}$ and their effect on lithium diffusion. Chem. Mater. 30 , 4995 (2018).

22. Zhang, W. et al. The detrimental effects of carbon additives in $\mathrm{Li}_{10} \mathrm{GeP}_{2} \mathrm{~S}_{12}$-based solid-state batteries. ACS Appl. Mater. Inter. 41, 35888 (2018). 
23. Wang C. et al. Manipulating interfacial nanostructure to achieve high-performance all-solid-state lithium-ion batteries. Small 3, 1900261 (2019).

24. Kim, K. \& Martin, S. W. Structures and properties of oxygen-substituted $\mathrm{Li}_{10} \mathrm{SiP}_{2} \mathrm{~S}_{12-\mathrm{x}} \mathrm{O}_{\mathrm{x}}$ solid-state electrolytes. Chem. Mater. 31, 3984-3991 (2019).

25. Banerjee, S., Zhang, X. \& Wang, L. Motif-based design of an oxysulfide class of lithium superionic conductors: Toward improved stability and record-high Li-ion conductivity. Chem. Mater. 31, 72657276 (2019).

26. Iwasaki, R. et al. Weak anisotropic lithium-ion conductivity in single crystals of $\mathrm{Li}_{10} \mathrm{GeP}_{2} \mathrm{~S}_{12}$. Chem. Mater. 31, 3694-3699 (2019).

27. Arico, A. S., Bruce, P., Scrosati, B., Tarascon, T. M. \& van Schalkwijk. Nanostructured materials for advanced energy conversion and storage devices. Nat. Mater. 4, 366-377 (2005).

28. Dawson, J. A. et al. Toward understanding the different influences of grain boundaries on ion transport in sulfide and oxide solid electrolytes. Chem. Mater. 31, 5296-5304 (2019).

29. Sakuda, A., Hayashi, A. \& Tatsumisago, M. Sulfide solid electrolyte with favorable mechanical property for all-solid-state lithium battery. Sci. Rep. 3, 2261 (2013).

30. Dawson, J. A., Canepa, P., Famprikis, T., Masquelier, C. \& Islam, M. S. Atomic-scale influence of grain boundaries on Li-ion conduction in solid electrolytes for all-solid-state batteries. J. Am. Chem. Soc. 140, 362-368 (2018).

31. Cheng, L. et al. Effect of surface microstructure on electrochemical performance of garnet solid electrolytes. ACS Appl. Mater. Inter. 7, 2073-2081 (2015).

32. Bai, L. et al. Interfacial ion-transport mechanism of $\mathrm{Li}_{7}\left(\mathrm{Al}_{0.1}\right) \mathrm{La}_{3} \mathrm{Zr}_{2} \mathrm{O}_{12}$ solid electrolyte modified by using a spark plasma sintering method. ChemElectroChem 5, 3918-3925 (2018).

33. Ma, C. et al. Atomic-scale origin of the large grain-boundary resistance in perovskite Li-ionconducting solid electrolytes. Energy Environ. Sci. 7, 1638-1642 (2014).

34. Ganapathy, S., Yu, C., van Eck, E. R. H. \& Wagemaker, M. Peeking across grain boundaries in a solid-state ionic conductor. ACS Energy Lett. 4, 1092-1097 (2019).

35. Yu, S. \& Siegel, D. J. Grain boundary contributions to Li-ion transport in the solid electrolyte $\mathrm{Li}_{7} \mathrm{La}_{3} \mathrm{Zr}_{2} \mathrm{O}_{12}$ (LLZO). Chem. Mater. 29, 9639-9647 (2017). 
36. Yu, C. et al. Unravelling Li-ion transport from picoseconds to seconds: Bulk versus interfaces in an argyrodite $\mathrm{Li}_{6} \mathrm{PS}_{5} \mathrm{Cl}-\mathrm{Li}_{2} \mathrm{~S}$ all-solid-state Li-ion battery. J. Am. Chem. Soc. 138, 11192-11201 (2016).

37. Canepa, P. et al. Particle morphology and lithium segregation to surfaces of the $\mathrm{Li}_{7} \mathrm{La}_{3} \mathrm{Zr}_{2} \mathrm{O}_{12}$ solid electrolyte. Chem. Mater. 30, 3019-3027 (2018).

38. Liu, Z. et al. Anomalous high ionic conductivity of nanoporous $\beta-\mathrm{Li}_{3} \mathrm{PS}_{4}$. J. Am. Chem. Soc. 135, 975-978 (2013).

39. Prutsch, D. et al. Nuclear spin relaxation in nanocrystalline $\beta-\mathrm{Li}_{3} \mathrm{PS}_{4}$ reveals low-dimensional $\mathrm{Li}$ diffusion in an isotropic matrix. Chem. Mater. 30, 7575-7586 (2018).

40. Tsukasaki, H., Mori, S, Morimoto, H., Hayashi, A. \& Tatsumisago, M. Direct observation of a noncrystalline state of $\mathrm{Li}_{2} \mathrm{~S}-\mathrm{P}_{2} \mathrm{~S}_{5}$ solid electrolytes. Sci. Rep. 7, 4142 (2017).

41. Zhang, J. et al. Flexible and ion-conducting membrane electrolytes for solid-state lithium batteries: Dispersion of garnet nanoparticles in insulating polyethylene oxide. Nano Energy 28, 447-454 (2016). 42. Yang, T., Gordon, Z. D., Li, Y. \& Chan, C. K. Nanostructured garnet-type solid electrolytes for lithium batteries: Electrospinning synthesis of $\mathrm{Li}_{7} \mathrm{La}_{3} \mathrm{Zr}_{2} \mathrm{O}_{12}$ nanowires and particle size-dependent phase transformation. J. Phys. Chem. C 119, 14947-14953 (2015).

43. Liu, X. et al. Facile synthesis of nanosized lithium-ion-conducting solid electrolyte $\mathrm{Li}_{1.4} \mathrm{Al}_{0.4} \mathrm{Ti}_{1.6}\left(\mathrm{PO}_{4}\right)_{3}$ and its mechanical nanocomposites with $\mathrm{LiMn}_{2} \mathrm{O}_{4}$ for enhanced cyclic performance in lithium ion batteries. ACS Appl. Mater. Inter. 9, 11696-11703 (2017).

44. Philip, M. A. et al. Improving cell resistance and cycle life with solvate-coated thiophosphate solid electrolytes in lithium batteries. ACS Appl. Mater. Inter. 11, 2014-2021 (2019).

45. Cao, Y. et al. A quasi-solid-state Li-S battery with high energy density, superior stability and safety. J. Mater. Chem. A 7, 6533-6542 (2019).

46. Mori, K. et al. Structural origin of massive improvement in Li-ion conductivity on transition from $\left(\mathrm{Li}_{2} \mathrm{~S}\right)_{5}\left(\mathrm{GeS}_{2}\right)\left(\mathrm{P}_{2} \mathrm{~S}_{5}\right)$ glass to $\mathrm{Li}_{10} \mathrm{GeP}_{2} \mathrm{~S}_{12}$ crystal. Solid State Ionics 301, 163-169 (2017).

47. Kahle, L., Musaelian, A., Marzari, N. \& Kozinsky, B. Unsupervised landmark analysis for jump detection in molecular dynamics simulations. Phys. Rev. Mater. 3, 55404 (2019).

48. Dawson, J. A., Chen, H. \& Islam, M. S. Composition screening of lithium-and sodium-rich antiperovskites for fast-conducting solid electrolytes. J. Phys. Chem. C 122, 23978 (2018). 
49. Kim, J.-S. et al. Atomistic assessments of lithium-ion conduction behavior in glass-ceramic lithium thiophosphates. ACS Appl. Mater. Inter. 11, 13 (2019).

50. Case, D. et al. Structure and ion transport of lithium-rich $\mathrm{Li}_{1+x} \mathrm{Al}_{x} \mathrm{Ti}_{2-x}\left(\mathrm{PO}_{4}\right)_{3}$ with $0.3<x<0.5$ : A combined computational and experimental study. Solid State Ionics 346, 115192 (2020).

51. Deng, Y. et al. Crystal structures, local atomic environments, and ion diffusion mechanisms of scandium-substituted sodium superionic conductor (NASICON) solid electrolytes. Chem. Mater. 30, 2618 (2018).

52. Deng, Y. et al. Enhancing the lithium ion conductivity in lithium superionic conductor (LISICON) solid electrolytes through a mixed polyanion effect. ACS Appl. Mater. Inter. 9, 7050 (2017).

53. Plimpton, S. Fast parallel algorithms for short-range molecular dynamics. J. Comput. Phys. 117, 1 (1995).

54. Jain, A. et al. Commentary: the materials project: a materials genome approach to accelerating materials innovation. APL Mater. 1, 011002 (2013).

55. He, X., Zhu, Y. \& Mo, Y. Origin of fast ion diffusion in super-ionic conductors. Nat. Comm. 8, 15893 (2017).

56. Evans, D. J. \& Holian, B. L. The Nose-Hoover thermostat. J. Chem. Phys. 83, 4069 (1985).

57. Gale, J. D. \& Rohl, A. L. The General Utility Lattice Program (GULP). Mol. Simul. 29, 291 (2003).

58. Tang, W., Sanville, E. \& Henkelman, G. A grid-based Bader analysis algorithm without lattice bias. J. Phys.: Condens. Matter 21, 084204 (2009).

59. Kresse, G. \& Furthmüller, J. Efficient iterative schemes for ab initio total-energy calculations using a plane-wave basis set. Phys. Rev. B 54, 11169 (1996).

60. Blöchl, P. E. Projector augmented-wave method. Phys. Rev. B 50, 17953 (1994).

61. Perdew, J. P. et al. Restoring the density-gradient expansion for exchange in solids and surfaces. Phys. Rev. Lett. 100, 136406 (2008).

62. Hirel, P. Atomsk: A tool for manipulating and converting atomic data files. Comput. Phys. Commun. 197, $212(2015)$. 